\title{
Resistive Micromegas for the Muon Spectrometer Upgrade of the ATLAS Experiment
}

\author{
Andreas Duedder on behalf of the ATLAS Muon Collaboration \\ Johannes Gutenberg-University Mainz, Mainz, Germany, \\ adudder@cern.ch
}

\begin{abstract}
In the context of the upgrade project of the ATLAS Muon system, we report on the construction of Micromegas detectors with an active area of 2 to $3 \mathrm{~m}^{2}$. The validation of the construction procedure is proven with results obtained with prototype detectors showing $100 \mu \mathrm{m}$ spatial resolution in the precision coordinate and above $98 \%$ efficiency.
\end{abstract}

Keywords: Resistive Micromegas, ATLAS, New Small Wheel, NSW

\section{Introduction}

The increase of the luminosity for the high luminosity LHC (HL-LHC) up to $7 \cdot 10^{34} \mathrm{~cm}^{-2} \mathrm{~s}^{-1}$ requires the upgrade of the most inner muon chambers in the forward region of the ATLAS detector, the so-called Small Wheel to grant an efficient operation of the full detector [1].

The New Small Wheel Upgrade project is based on Micromegas [2] and sTGC detectors [3]. Four different geometries of Micromegas chambers will be produced with a single chamber active area of 2 to $3 \mathrm{~m}^{2}$, summing up to a total active area of $1200 \mathrm{~m}^{2}$ in total (Figure 1). Each detector module consists of four independent readout layers as shown in Figure 2. The readout strips in two readout planes are parallel to the long side of the trapezoidal detector shape. These layers are dedicated to the reconstruction of the precision coordinate of the Micromegas chambers, the azimuthal coordinate in the ATLAS detector. The other two readout layers have strips, which are inclined by $\pm 1.5^{\circ}$ with respect to the two parallel layers to allow, in addition, the reconstruction of the second coordinate.

For the precision coordinate, a single plane resolution of better than $100 \mu \mathrm{m}$ is required to keep the $15 \% p_{\mathrm{T}}$ resolution of the ATLAS detector for $1 \mathrm{TeV}$ muon tracks and to cope with the environment in the ATLAS detector in the HL-LHC. Furthermore, the chambers have to be efficient up to an hit rate of $15 \mathrm{kHz} / \mathrm{cm}^{2}$ and to operate inside a magnetic field up to $0.3 \mathrm{~T}$. 


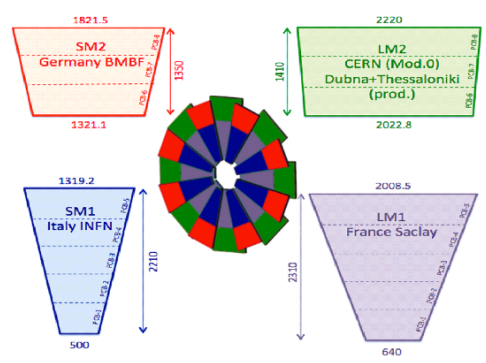

Fig. 1: Geometry of the ATLAS New Small Wheel Upgrade. The sizes of the different Micromegas modules and the distribution over the construction sites is shown (adapted from [4]).

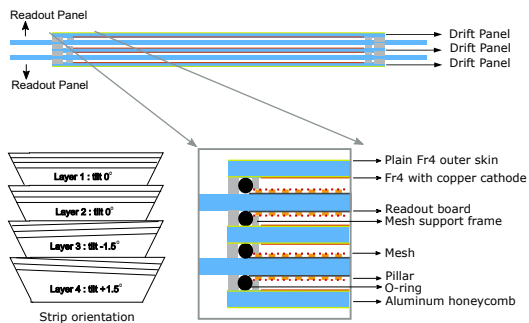

Fig. 2: Design of the New Small Wheel Micromegas quadruplet. The chamber is devided in 3 drift and 2 readout boards (adapted from [5]).

\section{Detector design}

Each Micromegas quadruplet consists of 5 panels (Figure 2), three so-called drift panels and two readout panels. All panels are sandwich structures of FR4 sheets on the outside, glued to an aluminium frame and stiffening aluminium honeycomb. On the FR4, the readout structure or a copper cathode is imprinted. The drift panels have a copper cathode on one side (outer panel) or both sides (inner panel) while the readout panels have imprinted readout strips with a resistive protection layer on both sides for spark protection and high rate capability.

Each panel side is build out of 3 to 5 single PCBs in a horizontal segmentation due to the mechanical limits in the PCB manufacturing. To achieve the required track resolution, the maximum displacement of the readout $\mathrm{PCB}$ within each layer and between different layers has to be less than $60 \mu \mathrm{m}$ and the allowed panel deformation from an ideal plane has to be below an RMS of $37 \mu \mathrm{m}$.

The readout panels are wider than the drift panels. The additional space hosts the readout electronics and an integrated cooling channel.

The micromesh is glued to a frame mounted to the drift panels, following the floating mesh approach. It is kept at a $5 \mathrm{~mm}$ distance from the readout plane by oval pillars.

\section{Panel Construction}

To match the very challenging requirements on the panels alignment and planarity, different approaches have been developed in the different construction sites for the construction of the panels. In all cases the basic idea is to transfer the planarity of a precision tooling (e.g. a granite table) to the panel. Therefore dedicated high planarity tables have been built. We distinguish between two main methods: the stiff back method and the vacuum bag method. In the stiff 
back method the PCBs are sucked to the precision surface during the gluing process. Dedicated high planarity tables have been constructed. The overall gluing process takes $48 \mathrm{~h}$ consisting in two sequential $24 \mathrm{~h}$ gluing steps, one per each face of the panel. The aluminium frames are glued to the PCBs which are aligned with respect to each other and the frames on a vacuum table or granite surface. After the curing, the workpiece is moved to a second moveable vacuum table. In the next step, a second PCB layer is aligned in the alignment structure used on the first day. The half finished workpiece, attached to the second vacuum table, is flipped over in the glue and aligned. This procedure guarantees the flatness of both gluing sides, since they are both attached to a precision surface. However, this approach needs two days for the gluing of one panel.

In contrast, the vacuum bag procedure allows for the gluing within $24 \mathrm{~h}$. Here the full sandwich is closed and aligned in one step and covered with a vacuum foil to press it against the precision surface. The planarity is transferred through the aluminium honeycomb and frames to the second surface. This implies more strict quality requirements for the internal parts, since their thickness variation is directly transferred to the upper PCB layer.

In most cases a combination of both approaches is used. The two day gluing process with a moveable vacuum table combined with a vacuum bag on the first gluing day ensures the flatness and extensive attachment of the frames and honeycomb.

For the alignment of the strips in the readout layer, two different solutions are in use as well. One is to provide precision holes for tight fitting pins in the PCBs and frames. In the second case, precision fences are glued under optical alignment surveillance to the boards. While the pins are inserted in holes or slots in the precision table, the fences are pushed against embedded reference points. In the later case, the drilling of the PCBs and frames is done after the panel gluing to avoid the masking of all holes during the gluing process itself.

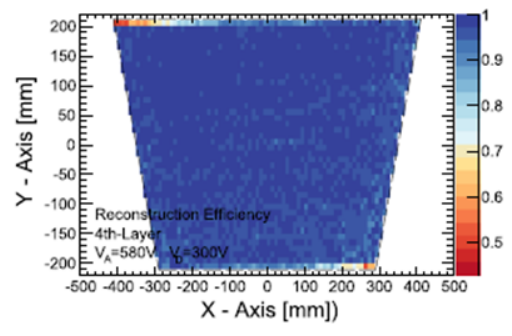

Fig. 3: Efficiency map of one inclined layer of the MMSW prototype chamber. The inefficiency in the upper left and lower right areas is due to the inclined strips, which leave these areas without strips and such inactive [5].

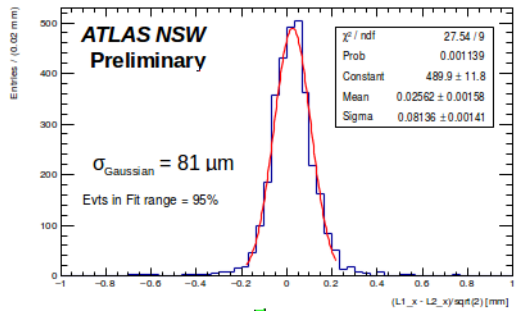

Fig. 4: Precision coordinate spacial resolution for the real size prototype chamber [4]. 


\section{Prototype Results}

The design and construction method underwent several test stages. The first stage with the trapezoidal detector geometry was the so-called MMSW detector. Two quadruplets have been constructed and tested [5]. Figure 3 shows the efficiency of the forth layer of one of the quadruplets. The efficiency is above $98 \%$ inside the active area of this layer. The efficiency of the other layer and the spatial resolution of both detector coordinates was found to be within the specifications.

The second stage of test was the construction of real size prototypes in the final geometry. The results for the resolution of the precision coordinate are presented in Figure 4. The data was taken with a $180 \mathrm{GeV}$ pion beam. In the histogram, the difference between the hit position in the two precision layer, corrected by the statistical factor $\sqrt{2}$ to retrieve the single plane resolution, was plotted. The resolution was estimated by an gaussian fit to $81 \mu \mathrm{m}$. The resolution in the second coordinate and the efficiency of this detector is also well inside the specification.

\section{Conclusion and Acknowledgement}

The production mechanism for the Micromegas chambers of the ATLAS NSW Upgrade project is well defined and under control. The results from prototype detectors indicate that the required performance parameters can be met and hence qualifiy the production process. The mass production has started in several construction sites allowing for the installation of the chambers during the next long shutdown period of the LHC starting in 2019.

We would like to thank Rui de Oliviera from the CERN PCB workshop for the close collaboration in the PCB manufacturing. Andreas Duedder was supported by the GRK Symmetry Breaking Graduate School.

\section{References}

[1] The ATLAS Collaboration: New Small Wheel Technical Design Report. CERNLHCC-2013-006. ATLAS-TDR-020 (2013)

[2] T. Alexopoulos et al.: A spark-resistant bulk-micromegas chamber for high-rate applications. Nuclear Instruments and Methods in Physics Research Section A, 640, p. $110(2011)$

[3] S.Majewski, G.Charpak, A.Breskin G.Mikenberg: A thin multiwire chamber operating in the high multiplication mode. Nuclear Instruments and Methods in Physics Research Section A, 217, p. 265-271 (1983)

[4] M. Iodice, Resistive Micromegas for the Muon Spectrometer Upgrade of the ATLAS Experiment. PoS(ICHEP2016)275 and Talk at ICHEP 2016

[5] Michele Bianco et al.: Construction of two large-size four-plane micromegas detectors. Nuclear Instruments and Methods in Physics Research Section A 814, 117-130 (2016) 